\title{
GENETIC COMPLEXITY OF PLASMODIUM FALCIPARUM GAMETOCYTES ISOLATED FROM THE PERIPHERAL BLOOD OF TREATED GAMBIAN CHILDREN
}

\author{
COLIN J. SUTHERLAND, ALI ALLOUECHE, LOUISA McROBERT, ROSALYNN ORD, JAMIE LEGGAT, \\ GEORGES SNOUNOU, MARGARET PINDER, AND GEOFFREY A. T. TARGETT \\ Department of Infectious and Tropical Diseases, London School of Hygiene and Tropical Medicine, London, United Kingdom; \\ Medical Research Council Laboratories, Banjul, The Gambia; Unité Parasitologie Bio-Médicale, Institut Pasteur, Paris, France
}

\begin{abstract}
The genetic complexity of Plasmodium falciparum gametocytes isolated from Gambian children participating in a controlled trial of anti-malarial therapy was investigated. RNA and DNA were prepared from gametocytepositive blood, which was also used in transmission experiments with Anopheles gambiae mosquitoes. Amplification by a reverse transcriptase-polymerase chain reaction (RT-PCR) of transcripts from the genes for the ring-infected erythrocyte surface antigen and the 16-kD antigen, which exhibit asexual and sexual stage-specific expression, was used to identify 30 post-treatment gametocyte isolates in which trophozoites persisted below the threshold of detection by microscopy. These included isolates from children who received sulfadoxine/pyrimethamine plus artesunate. Twentynine gametocyte-positive isolates that were free of subpatent trophozoites were examined further by PCR amplification of polymorphic genomic loci. We estimate that an average minimum of 2.3 genotypes occurred in these gametocyte-only isolates, and many of these were shown to be infective to mosquitoes. Thus, meiotic recombination between different genotypes is predicted to be a common event in this study area.
\end{abstract}

\section{INTRODUCTION}

The gametocytes (sexual stages) of malaria parasites are the essential link between human blood-stage infection and the mosquito-borne phase of the parasite life cycle. In the case of Plasmodium falciparum, an acute clinical attack is frequently followed by a wave of gametocytes in the peripheral blood after 6-10 days, ${ }^{1}$ even when treated. ${ }^{2,3}$ This is in contrast to asymptomatic and chronic infections in semiimmune people, which are associated with recurrent, lowdensity, intermittent gametocytemia. ${ }^{4,5}$ In acute episodes of falciparum malaria, the delay between peak asexual parasitemia and the appearance of mature gametocytes in the periphery reflects a period of sequestered development of gametocytes in bone marrow, spleen, and probably a number of other organs. ${ }^{6,7}$

In natural populations of Plasmodium, meiotic recombination between different genotypes can occur when the mosquito blood meal contains a mixture of genetically distinct gametocytes. Only from such mixtures can diploid zygotes capable of generating reassorted genotypes in the haploid progeny (sporozoites) be formed. In contrast, a blood meal that contains gametocytes of a single genotype can only result in self-fertilization, where the generation of diversity will be restricted to limited recombination among the sub-telomeres of heterologous chromosomes. ${ }^{8}$ To address the controversial possibility that $P$. falciparum exhibits a clonal population structure, ${ }^{9}$ the degree of self-fertilization has previously been examined by genotyping oocysts in wild-caught infected mosquitoes. ${ }^{10,11}$ However, attempts to determine directly the number of genotypes present as circulating gametocytes using standard approaches are significantly hampered by the concomitant presence of circulating asexual parasites in most gametocyte-positive hosts.

We have recently shown in controlled trials in The Gambia that children with clinical malaria are more likely to become gametocytemic after treatment with sulfadoxine/ pyrimethamine (SP, Fansidar ${ }^{\circledR}$; F. Hoffmann-La Roche, Basel, Switzerland) than after treatment with either chloroquine (CQ) or with a combination of SP and artesunate. ${ }^{3}$ Gametocyte densities after treatment were highest among children in the SP group, and lowest after treatment with SP plus artesunate. Parasites present in venous blood taken from some gametocyte-positive children from each treatment group were able to infect Anopheles gambiae mosquitoes by membrane-feeding, showing that the emergence of viable gametocytes was not completely prevented by any of the treatments tested. The majority of post-treatment gametocyte carriers in this study did not carry patent trophozoites (i.e., ringstage parasites), and so we surmised that these blood samples would be a good source of gametocyte-derived genetic material free of nucleic acids from asexual parasites.

We extracted RNA and DNA from the venous blood of 109 gametocyte-positive treated children who donated blood for transmission experiments. These nucleic acid samples were used as templates for the amplification of a number of parasite genes. To exclude those samples with microscopically subpatent asexual parasites, and to confirm the presence of gametocytes, we carried out amplification by a reverse transcriptase-polymerase chain reaction (RT-PCR) of stagespecific transcribed sequences. We successfully amplified gametocyte-specific transcripts in 65 samples and, in half of those samples, also detected transcripts from subpatent trophozoites that had survived drug treatment. We could therefore select gametocyte-positive, trophozoite-negative isolates in which the numbers of genotypes present were measured. Our findings have implications for estimates of meiotic recombination frequency in our population, and further our understanding of the pattern of malaria transmission in this endemic African setting.

\section{MATERIALS AND METHODS}

Study subjects. The parasite isolates used in this study were obtained from patients participating in a clinical trial that was conducted with full ethical approval from both the London School of Hygiene and Tropical Medicine ethical committee, and the joint Gambian Government/Medical Research Council ethics committee. Documented informed consent was obtained from parents or guardian.

Five hundred and ninety-nine Gambian children with uncomplicated clinical malaria who presented to Farafenni 
Health Center between September and December 1998 were treated with one of three treatment regimens: CQ $(n=135)$, SP $(n=277)$, and SP plus artesunate $(n=187)$ as previously described. ${ }^{3}$ During follow-up, a subject was considered gametocyte-negative if no sexual-stage parasites were observed in Giemsa-stained thick blood smears per 1,000 leukocytes. A subject was considered trophozoite-negative if no ring-stage parasites were observed per 200 leukocytes. Parasite density was calculated assuming 8,000 leukocytes/ $\mu$ L. The 109 children participating in this study were gametocyte-positive during follow-up (92 on day 4, 14 on day 6 or 7, and one each on days 8,10 , and 16). This group was composed of 55 children between one and five years old, 38 children between six and 10 years old, and 16 children between 11 and 18 years old. For each of these children, a parent's or guardian's consent was obtained for $3 \mathrm{~mL}$ of venous blood to be taken for transmission studies. The methods used for transmission have been described. $^{3}$

Samples and nucleic acid extraction. An aliquot of 50-300 $\mu \mathrm{L}$ of fresh venous blood was frozen at $-20^{\circ} \mathrm{C}$, without any additives, immediately after transmission experiments with $A$. gambiae. ${ }^{3}$ A few months later, blood was thawed and $30 \mu \mathrm{L}$ was immediately added to $1,000 \mu \mathrm{L}$ of TRI Reagent (Sigma, Poole, Dorset, United Kingdom). After thorough mixing, and a brief incubation at room temperature, these samples were stored at $-20^{\circ} \mathrm{C}$ prior to being shipped to London. DNA was extracted from the remainder of the thawed blood using the Nucleon BCC kit (Nucleon Biosciences, Coatbridge, Scotland), which is specifically designed for extracting DNA from blood samples. To remove contaminating material due to blood clotting in many samples, an extra extraction with phenol/chloroform/isoamyl alcohol (50:49:1) was performed following extraction, followed by precipitation with ethanol. The pellet was washed briefly in $75 \%$ ethanol, and resuspended in $40 \mu \mathrm{L}$ of nuclease-free water. For pre-treatment isolates, DNA was extracted from dried blood spots on GFC filter paper, as described previously. ${ }^{12}$

Total RNA was extracted from the blood samples stored in TRI reagent according to the manufacturer's instructions. The final RNA pellet was resuspended directly into a deoxynuclease reaction (RQ RNAse-free DNAse; Promega, Southampton, United Kingdom) in a total volume of $50 \mu \mathrm{L}$ that included approximately 50 units of nuclease and $1 \mu \mathrm{g}$ of yeast tRNA (Sigma). After incubation for $1-2$ hours at $37^{\circ} \mathrm{C}$, the reaction was stopped with an EGTA solution supplied with the DNAse enzyme. The sample was then diluted to a volume of $100 \mu \mathrm{L}$ with water and $3 \mathrm{M}$ sodium acetate was added to give a final concentration of $0.3 \mathrm{M}$. RNA was precipitated by the addition of $250 \mu \mathrm{L}$ of $100 \%$ ethanol and the sample was placed at $-20^{\circ} \mathrm{C}$ for at least two hours. The RNA was recovered by centrifuging the sample at $12,000 \times g$ for 20 minutes. The pellet was then washed briefly in $75 \%$ ethanol, air-dried, and resuspended in $20 \mu \mathrm{L}$ of nuclease-free water (Promega). Subsequent RT-PCR experiments showed evidence of residual DNA contamination in some of our RNA samples. These were incubated with DNAse and the restriction enzyme Rsa I for one hour at $37^{\circ} \mathrm{C}$ prior to subsequent RT-PCR experiments.

Amplification of nucleic acids. All RT-PCRs were performed with the Access-RT-PCR System (Promega), which uses Tth I thermostable polymerase. This enzyme shows excellent activity in buffers suitable for the avian myeloblastosis virus (AMV) reverse transcriptase (RTase). Procedures provided with the kit were followed and a single program on a thermal cycler (Omnigene; Hybaid, Ashford, UK) was used. Briefly, for the duplexed RT-PCRs for the asexual-specific ring-infected erythrocyte surface antigen ( $r e s a)$ and the sexual stage-specific $16-\mathrm{kD}$ antigen $(p f s 16)$, the four outer primers (final concentration $=250 \mathrm{nM}$ ), all reagents, and both enzymes (Tth I and AMV-RTase) were mixed in each tube. All test samples were accompanied by an identical sample lacking RTase as a control for DNA contamination in the RNA samples. All tubes were incubated at $48^{\circ} \mathrm{C}$ for 45 minutes to permit first-strand cDNA synthesis. The temperature was then increased to $95^{\circ} \mathrm{C}$ for five minutes to denature the RTase, followed by 45 cycles of amplification. Each cycle was $94^{\circ} \mathrm{C}$ for 30 seconds, $48^{\circ} \mathrm{C}$ for one minute, and $65^{\circ} \mathrm{C}$ for one minute. One microliter of the RT-PCR product was used as a template in two nested PCRs (volume $=25 \mu \mathrm{L}$ for each PCR). Samples were amplified for 30 cycles with annealing temperatures of $62^{\circ} \mathrm{C}$ and $59^{\circ} \mathrm{C}$ for resa and $p f s 16$, respectively. Products were visualized by electrophoresis on $1.5 \%$ agarose gels in Tris-borate EDTA buffer. The gels were stained with ethidium bromide and photographed over a UV transilluminator.

Amplification of merozoite surface protein 1 ( $m s p-1)$ and $m s p-2$ sequences was performed exactly as previously described $^{12}$ using $1 \mu \mathrm{L}$ of prepared DNA in the first multiplex reaction (with both outer primer sets) and $1 \mu \mathrm{L}$ of these products in subsequent, allelic, family-specific nested PCRs. Amplification products were visualized by electrophoresis on $2 \%$ MetaPhor $^{\circledR}$ (FMC BioProducts, Rockland, ME) agarose gels. The gels were stained with ethidium bromide, photographed as described earlier, and scored by two independent observers.

Oligonucleotide primers. The following oligonucleotide primers for amplification of nucleic acids were designed for this study: pfs16 outer PCR: CSo53 caacatgaatattcgaaagttc, CSo54 ttaagaatcatcatctccttcgtctcc; pfs 16 inner PCR: CSo72 ttcttcgcttttgcaaacctgg, CSo73 tttatcatcatctgcgttcttcg; resa outer PCR: CSo83 aaaagaaatgaaaataagagc, CSo85 gttttcga/ gagcggtaaac; resa inner PCR: CSo86 gttgtgttctaaacgtggtgttc, CSo87 cagcatatgggtttactggc. Primers for amplification of $m s p-1$ and $m s p-2$ sequences have been described elsewhere. ${ }^{12}$

Statistical analysis. Statistical significance was examined with Student's $t$-test for continuous data and the chi-square test for categorical variables using Stata 6.0 software (Stata Corp, College Station, TX).

\section{RESULTS}

Amplification of transcripts from the peripheral blood of
gametocyte-positive children. The RT-PCR amplification of transcripts from $p f s 16$, which encodes the $16-\mathrm{kD}$ sexual stagespecific antigen, ${ }^{13,14}$ and resa, which encodes the asexualspecific ring-infected erythrocyte surface antigen, ${ }^{15}$ was attempted with 109 RNA samples from gametocyte-positive children. ${ }^{3}$ The purpose of this was to determine if any of the RNA samples were purified from the peripheral blood of patients that had harbored subpatent trophozoites in addition to gametocytes. Interpretable results, free of DNA-derived amplification products, were obtained from 99 RNA samples for $p f s 16$ and 100 RNA samples for resa. For $p f s 16,64$ of the 
99 RNA samples were positive in the RT-PCR. Since all samples tested were gametocyte-positive by microscopy, we regarded $p f s 16$-negative samples as RT-PCR failures, probably due to RNA degradation during storage or sample extraction, and excluded them from subsequent analyses. Such failure of $p f_{s} 16$ amplification was significantly more common among RNA samples from children with gametocyte densities $\leq 40 / \mu \mathrm{L}(\mathrm{n}=49)$ compared with those with gametocyte densities $>40 / \mu \mathrm{L}\left(\mathrm{n}=50 ; \chi^{2}=13.3109\right.$, degrees of freedom [df] $=1, P<0.001$ ). Most (30 of 34) of these $p f s 16$-negative samples were also resa-negative, which suggests that RNA quality or concentration is a critical factor in amplification failures.

An interpretable RT-PCR result for resa was obtained from 60 of the $p f s 16$-positive RNA samples. Thirty of these 60 RNA samples were resa-positive: 14 from SP-treated children, 11 from SP plus artesunate-treated children, and five from CQ-treated children. Only three of these resa-positive RNA samples were from children with patent trophozoites at the time of sampling. Thus, either subpatent trophozoites persisted in the 27 remaining individuals, or gametocytes also transcribe resa. If the latter were true, we would be more likely to detect resa in samples with high gametocyte densities. However, there was no significant association between isolates having gametocyte densities $>40 / \mu l(n=50)$ and the RNA from those isolates that were resa-positive by the RTPCR $\left(\chi^{2}=2.102\right.$, df $\left.=1, P=0.147\right)$. We conclude that the 30 samples that were $p f s 16$-positive and resa-negative were composed of only gametocytes. Twenty-five of these were obtained four days after treatment, and the remainder were obtained 7-16 days after treatment.

Comparison of the multiplicity of infection in gametocyte samples with pre-treatment samples. Use of the nested PCR amplification and fractionation of products by electrophoresis on agarose gels resulted in the successful amplification of sequences at the $m s p-1$ and $m s p$ - 2 loci for both pre-treatment and follow-up DNA samples from 59 of the 60 gametocytepositive isolates described earlier. Twenty-nine of these 59 gametocyte-positive isolates were resa-negative. We sought to determine the number of parasite genotypes represented in each by counting the number of distinct amplified products from both loci. The greater of the two numbers, from either $m s p-1$ or $m s p-2$, was our estimate of the minimum number of genotypes ( $\mathrm{MNG})$.

Estimates of MNG were first derived from DNA samples extracted from parasite isolates taken prior to treatment from the 59 children who later became gametocyte-positive. These samples represent the parasite population associated with each child's clinical attack, and therefore are composed of trophozoites at a high density. In 15 of the isolates from which these samples were derived, gametocytes were also detected by microscopy prior to treatment. A frequency histogram of estimated MNG for these 59 samples is shown in Figure 1.

The MNG was then estimated for each of the corresponding 59 post-treatment gametocyte isolates used for mosquito infection studies (Figure 2). Significantly fewer resa-positive isolates were monoclonal (single genotype) compared with resa-negative (gametocyte only) isolates $\left(\chi^{2}=7.95, \mathrm{df}=1, P\right.$ $=0.005)$ and resa-negative gametocyte samples were generally composed of fewer distinct parasite genotypes (Figure 2). Nevertheless, the majority of gametocyte-only isolates contained two or more genotypes, and the mean MNG for these

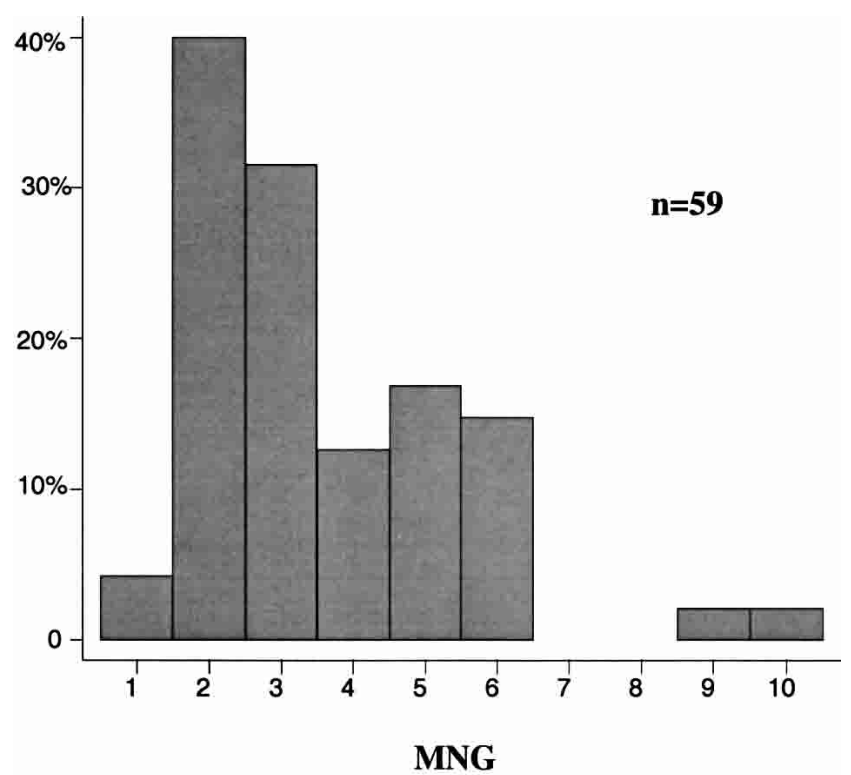

\section{Mean MNG: $3.56(95 \% \mathrm{CI}+/-0.48)$}

FIGURE 1. Frequency histogram of estimated minimum number of genotypes (MNG) detected among the pre-treatment infections of 59 children who were later positive for gametocytes.

29 isolates was greater than two, indicating that mosquitoes feeding on these hosts would frequently ingest genetically heterogeneous gametocyte mixtures. The number of multiple-genotype gametocyte isolates in the three treatment groups were as follows: for $\mathrm{CQ}$, one isolate $(\mathrm{n}=3)$, for $\mathrm{SP}$, nine isolates $(\mathrm{n}=14)$, and for SP plus artesunate, eight isolates $(\mathrm{n}=12)$. There was no association between MNG of the gametocyte samples and age.

As might be expected, the average number of genotypes in pre-treatment isolates was higher than among post-treatment gametocyte-positive isolates. There was no difference in the pre-treatment MNG between those subjects with detectable resa transcripts in follow-up and those without these transcripts. Analysis of paired samples from each individual showed that the reduction in MNG after treatment was statistically significant $(P<0.001$, by one-sided paired $t$-test).

We have shown that an average of one less genotype is present in post-treatment gametocyte-positive isolates than was present in the preceding clinical infection. Can these persisting genotypes be matched with genotypes seen prior to treatment? A conservative criterion by which one can judge differences between isolates is to count distinct amplified bands in each of the five allelic families (three for $m s p-1$ and two for $m s p$-2) among the gel-fractionated PCR products. An increase in the number of bands for a particular allelic family indicates that the follow-up isolate includes a genotype not detected in the pre-treatment isolate. This approach ignores fragment size as a criterion; including size would add to our discriminating power, but would still underestimate both genotype number and the number of genotypic differences between paired isolates. Electrophoresis results for pretreatment and post-treatment samples from all children $(\mathrm{n}=$ 10 ) in whom additional products were observed are shown in Table 1.

All 10 of these individuals, in which there is evidence of the 


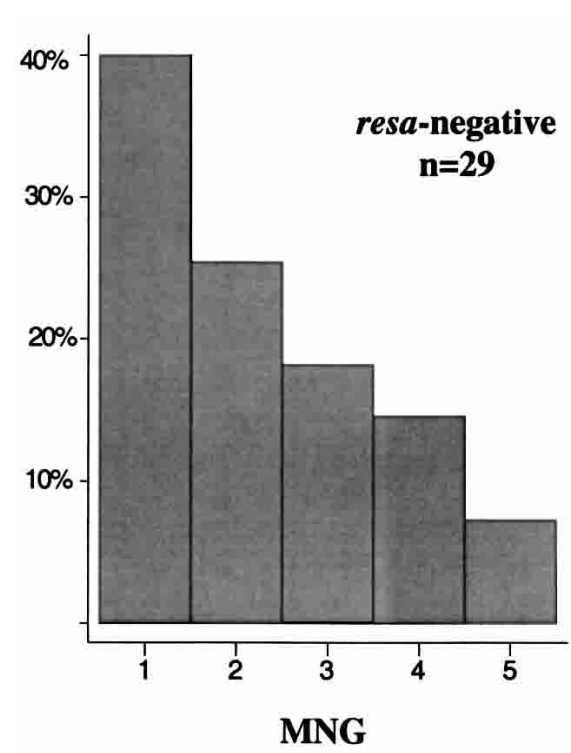

Mean MNG: $2.28(95 \% \mathrm{CI}+/-0.50)$ 2-sided t-test: $\quad \mathbf{P}=\mathbf{0 . 0 9 7 4}$

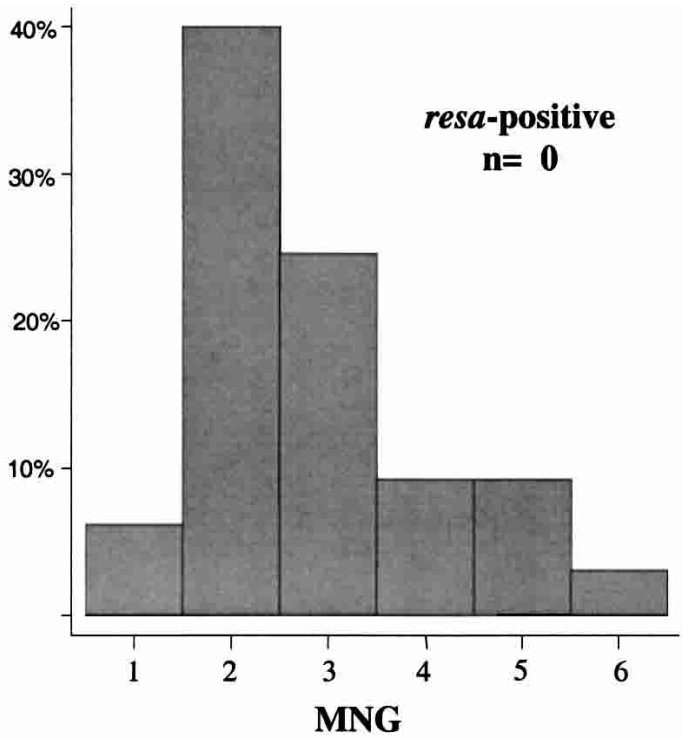

Mean MNG: $\quad 2.83(95 \% C I+/-0.46)$

FIGURE 2. Frequency histogram of estimated minimum number of genotypes (MNG) detected among both ring-infected erythrocyte surface antigen (resa)-negative (left) and resa-positive post-treatment infections from the same 59 gametocyte-positive children as in Figure 1.

presence of at least one additional genotype in the posttreatment sample, were resa-negative by the RT-PCR. This means that the appearance of additional genotypes in a gametocyte sample is significantly associated with the absence of persistent trophozoites $\left(\chi^{2}=12.0, P=0.001\right)$. The appearance of additional genotypes was not associated with the estimated MNG in the gametocyte isolate or with the day of follow-up or treatment group.

MNG and infectivity to mosquitoes. We have shown that gametocytes isolated from Gambian children after treatment were frequently composed of two or more genotypes. It was necessary to show that multiple genotype gametocyte isolates

TABLE 1

Number of bands detected by electrophoresis for each of the allelic families of merozoite surface protein-1 (msp-1) $(\mathrm{K}, \mathrm{M}$, and R) and $m s p-2$ (F and I) in pre-treatment and post-treatment gametocyte samples from 10 individuals in which new bands appeared in the gametocyte-positive follow-up sample*

\begin{tabular}{|c|c|c|c|c|c|c|c|c|c|c|}
\hline & \multicolumn{10}{|c|}{ Number of products } \\
\hline & \multicolumn{5}{|c|}{ Pre-treatment isolates } & \multicolumn{5}{|c|}{ Post-treatment gametocyte isolates } \\
\hline & $\mathrm{K}$ & M & $\mathrm{R}$ & $\mathrm{F}$ & I & $\mathrm{K}$ & M & $\mathrm{R}$ & $\mathrm{F}$ & I \\
\hline Patient 1 & 0 & 1 & 0 & 1 & 1 & $\mathbf{1}$ & 1 & $\mathbf{1}$ & 2 & 1 \\
\hline 2 & 1 & 1 & 0 & 0 & 3 & 2 & 1 & 0 & $\mathbf{1}$ & 1 \\
\hline 3 & 2 & 0 & 0 & 5 & 0 & 1 & 0 & 0 & 2 & 1 \\
\hline 4 & 0 & 0 & 1 & 3 & 2 & 1 & 0 & 1 & 1 & 1 \\
\hline 5 & 1 & 0 & 0 & 0 & 2 & 1 & 0 & 0 & 1 & 1 \\
\hline 6 & 1 & 0 & 0 & 1 & 1 & 1 & 0 & 1 & 1 & 0 \\
\hline 7 & 1 & 1 & 0 & 0 & 1 & 1 & 0 & 0 & 1 & 0 \\
\hline 8 & 4 & 0 & 1 & 0 & 3 & 4 & 0 & 1 & 2 & 1 \\
\hline 9 & 1 & 0 & 0 & 6 & 0 & 0 & 0 & 0 & 3 & 1 \\
\hline 10 & 1 & 0 & 1 & 0 & 0 & 0 & 0 & 1 & 1 & 0 \\
\hline
\end{tabular}

are at least equally likely to infect $A$. gambiae to demonstrate that mating between genetically different gametocytes is likely. The relationship between crude infectivity, determined for each child in our transmission study, ${ }^{3}$ and monoclonality of gametocyte samples is shown in Table 2 .

Multiple-genotype gametocyte isolates were similar in infectivity to monoclonal isolates. This finding is corroborated by comparison of the mean MNG among infective and noninfective isolates: $2.7 \pm$ genotypes $(95 \% \mathrm{CI} \pm 0.54 ; \mathrm{n}=32)$ and $2.4 \pm 0.40(\mathrm{n}=27)$ genotypes $(\mathrm{P}=0.31$, by two-sided t-test).

\section{DISCUSSION}

We have performed a genotypic and phenotypic survey of gametocyte-positive $P$. falciparum isolates from Gambian children who had received anti-malarial treatment between four and 16 days earlier. Each of these isolates was also used in transmission experiments with membrane-fed $A$. gambiae mosquitoes. ${ }^{3}$ Our data show that four days after treatment with CQ, SP, or SP plus artesunate subpatent trophozoites can be detected by an RT-PCR in a considerable proportion

TABLE 2

Relationship between the estimated minimum number of genotypes (MNG) in a gametocyte sample and the crude infectivity of that sample to mosquitoes*

\begin{tabular}{cccr}
\hline \multirow{2}{*}{ Any mosquitoes infected? } & \multicolumn{2}{c}{ Number of gametocyte-positive isolates with } & \\
\cline { 2 - 3 } & MNG $=1$ & MNG $>1$ & Total \\
\hline No & $5(38.5 \%)$ & $23(48.9 \%)$ & 28 \\
Yes & $8(61.5 \%)$ & $24(51.1 \%)$ & 32 \\
Total & $13(21.7 \%)$ & $47(78.3 \%)$ & 60 \\
\hline$* \chi^{2}=0.4489 ; P=0.503$. & &
\end{tabular}


of isolates. The mean minimum estimate of the number of parasite genotypes in those isolates that consisted of gametocytes only was found to be 2.3 genotypes. Since many of the polyclonal isolates were shown to be infective to mosquitoes, we conclude that cross-fertilization events between two different genotypes, and thus the potential conditions for meiotic recombination, commonly occur in mosquitoes feeding on treated children. For example, $25.6 \%$ of SP-treated children are infectious seven days after treatment, ${ }^{3}$ and nine of 14 gametocyte isolates from the SP group contained multiple parasite genotypes. Thus, approximately $16 \%$ of the children treated with SP will be infective to mosquitoes and may be expected to transmit a mixture of genetically distinct gametocytes.

A relatively large number of $p f s 16$ RT-PCR failures (34 of $109,31.2 \%$ ) occurred among our gametocyte-positive isolates with the methods we used. There was a tendency for the RT-PCR to fail when gametocytes occurred at low density, but this did not account for all failures. Many of our samples were stored between one and two years in TRI reagent at $-20^{\circ} \mathrm{C}$, and Kyes and others, ${ }^{16}$ using similar methods, have shown that long-term storage above $-70^{\circ} \mathrm{C}$ reduces the yield of RNA. In future studies, we need to improve our sample storage methods, and process stored material sooner.

Of the 60 pfs 16-positive RNA samples studied in detail, no fewer than 30 were also positive for resa transcripts. We believe that resa transcription in gametocytes can be ruled out due to the lack of association between resa positivity and gametocyte density, assuming that there are no particular subsets of circulating gametocytes that do transcribe the resa gene. We therefore conclude that in half of our isolates gametocytes co-exist with trophozoites that are too scarce to be observed on thick blood films.

This is the first time to our knowledge that an RT-PCR has been used to detect stage-specific parasite survival after drug treatment. A number of these isolates occurred in children who had received SP plus artesunate (both one-day and three-day regimens), confirming that in vivo, artesunate did not radically clear asexual parasites in all patients in our study population. We do not believe that the day 4 parasites are resistant to artesunate, but rather that there is a pharmacodynamic tail of persisting sensitive parasites in some treated individuals. These may be drug-damaged parasites unable to multiply further, as have been observed in P. chabaudiinfected mice treated with pyrimethamine. ${ }^{17}$ However, given that artesunate has a very short half-life and will have been exhausted in the circulation by day 4 , we favor the interpretation that these are fully viable parasites, capable of recrudescence. This phenomenon does have serious implications for combination therapy that pairs artesunate with a vulnerable drug, such as CQ or SP, for which resistance already occurs in the population concerned. There is then a finite likelihood of parasites intrinsically resistant to the vulnerable drug being among those that escape artesunate in the pharmacodynamic tail. Such parasites could cause delayed recrudescences among patients receiving combination therapy. We have evidence that late treatment failures do indeed occur with the combination of CQ plus artesunate in The Gambia (Sutherland CJ and others, unpublished data), illustrating the importance of combining artesunate with drugs known to be highly effective. ${ }^{18}$

The key finding of our study is that gametocytes ingested by mosquitoes feeding on this population are likely to form heterodiploid zygotes, and thus generate sporozoites with novel genotypes following meiotic recombination. Whereas other mechanisms of recombination are thought to contribute to genetic heterogeneity in Plasmodium, particularly adjacent to telomeres, ${ }^{8,19}$ meiotic recombination is the most important source of gene reassortment. No doubt this has been a fundamental driving force behind the high level of genetic diversity that characterizes African parasite populations. ${ }^{10,20}$ Elsewhere, in parasite populations with limited genetic diversity, self-fertilization may be a far more frequent event and this can result in a population structure approaching clonality. ${ }^{21}$

These findings concerning the occurrence of multiple genotypes among gametocytes circulating at a given time cannot be automatically extrapolated to all gametocyte carriers in the Gambian host population. Since our study was conducted among children who had been treated for a clinical malaria attack, gametocytes found four days after treatment represent the beginning of the gametocyte peak commonly observed following clinical falciparum malaria in non- or semi-immune people. ${ }^{1-3}$ However, many of the gametocytemic people in the study area at any one time will have had asymptomatic infections and will not have sought treatment. Furthermore, many of them will be adults who tend to have well-developed immunity and carry low density gametocytemia for extended periods. ${ }^{5}$ Thus, it is difficult to estimate the relative contribution of treated children to the overall pool of transmissible parasites. For this reason, our results should be seen as a genotypic and phenotypic description of infective gametocytes from one important source: treated children. Complementary studies of treated and untreated asymptomatic gametocyte-carriers currently being undertaken in our study area should allow us to describe the genetic characteristics of gametocytes from other sources.

A recently established paradigm depicts $P$. falciparum infections in African people as complex mixtures of multiple parasite genotypes with intricate dynamics. ${ }^{2-24}$ Four days after treatment, any remaining asexual parasites represent the dwindling tail of the clinical peak of parasitemia, and the observed MNG in these samples reflects the MNG of the clinical peak. Circulating gametocytes, in contrast, must have differentiated from their asexual progenitors at least three days before treatment because their development takes 7-10 days. These progenitors may have been genetically different from the trophozoites that make up the clinical peak of parasitemia, as will be the gametocytes derived from them. In addition, we identified 10 gametocyte isolates in which novel allelic variants, distinct from any observed before treatment, were detected: each of these was resa-negative by the RTPCR. The genotype of a follow-up sample is therefore more likely to differ from that of the pre-treatment parasite population if all trophozoites have been eliminated, suggesting that in the absence of treatment gametocytes often co-exist with asexual parasites that are of a different genotype. This has implications for anti-gametocyte immunity, and Hayward and others ${ }^{25}$ have suggested that early developing gametocytes in vitro share immunodominant antigens of the $P$. falciparum erythroctye membrane protein 1 with their trophozoite progenitors.

In summary, we have used a combination of longitudinal genotyping and an RT-PCR to determine the genetic complexity of gametocytes circulating in the peripheral blood of 
treated Gambian children who had presented with clinical malaria. We have been able to show that among gametocyte isolates without detectable trophozoites there is on average more than two distinct genotypes present. Thus, conditions for frequent meiotic recombination are met.

Acknowledgments: We thank the staff of the Medical Research Council Laboratories in Farafenni and Fajara, The Gambia, and the management and staff of the AFPRC Hospital in Farafenni for their invaluable contribution to this work. We also acknowledge the support of Lorenz von Seidlein, Musa Jawara, Gijs Walraven, and Keith McAdam, and thank the participating children and their parents and guardians.

Financial support: This study was supported by Wellcome Trust grant \#047250 awarded to Geoffrey A. T. Targett, Wellcome Trust grant \#061910 awarded to Colin J. Sutherland, and the European Commission project QLRT-PL1999-0075.

Authors' addresses: Colin J. Sutherland, Ali Alloueche, Louisa McRobert, Rosalynn Ord, Jamie Leggat, and Geoffrey A. T. Targett, Department of Infectious and Tropical Diseases, London School of Hygiene and Tropical Medicine, Keppel Street, London WC1E 7HT, United Kingdom, Telephone: 44-20-7636-8636, Fax: 44-20-7636-8739. Georges Snounou, Unité Parasitologie Bio-Médicale, Institut Pasteur, 25-28 rue du Dr. Roux, 75015 Paris France, Telephone: 33-0140-61-37-35, Fax: 33-01-45-68-86-40. Margaret Pinder Medical Reseach Council Laboratories, PO Box 273, Banjul, The Gambia, Telephone/Fax: 220-495-917.

Reprint requests: Colin J. Sutherland, Immunology Unit, Department of Infectious and Tropical Diseases, London School of Hygiene and Tropical Medicine, Keppel Street, London WC1E 7HT, United Kingdom, Telephone: 44-20-7927-2338, Fax: 44-20-7636-8739, E-mail: colin.sutherland@1shtm.ac.uk.

\section{REFERENCES}

1. Shute PG, Maryon M, 1951. A study of gametocytes in a West African strain of Plasmodium falciparum. Trans $R$ Soc Trop Med Hyg 44: 421-438.

2. Robert V, Awono-Ambene HP, Le Hesran J-Y, Trape J-F, 2000. Gametocytemia and infectivity to mosquitoes of patients with uncomplicated Plasmodium falciparum malaria attacks treated with chloroquine or sulfadoxine plus pyrimethamine. Am J Trop Med Hyg 62: 210-216.

3. Targett G, Drakeley C, Jawara M, von Seidlein L, Coleman R, Deen J, Pinder M, Doherty T, Sutherland C, Walraven G, Milligan P, 2001. Artesunate reduces but does not prevent posttreatment transmission of Plasmodium falciparum to Anopheles gambiae. J Infect Dis 183: 1254-1259.

4. Babiker HA, Abdel-Wahab A, Ahmed S, Suleiman S, RanfordCartwright LC, Carter R, Walliker D, 1999. Detection of low level Plasmodium falciparum gametocytes using reverse transcriptase polymerase chain reaction. Mol Biochem Parasitol 99: 143-148.

5. Drakeley CJ, Flobbe K, Greenwood BM, Targett GAT, 2000. Plasmodium falciparum gametocytes in Gambian adults. Ann Trop Med Parasitol 94: 399-401.

6. Smalley ME, Abdalla S, Brown J, 1980. The distribution of Plasmodium falciparum in the peripheral blood and bone marrow of Gambian children. Trans R Soc Trop Med Hyg 75: 103-105.

7. Rogers NJ, Hall BS, Obiero J, Targett GAT, Sutherland CJ, 2000. A model for sequestration of the transmission stages of Plasmodium falciparum: adhesion of gametocyte-infected erythrocytes to human bone-marrow cells. Infect Immun 68 : 3455-3462.

8. Freitas-Junior LH, Bottius E, Pirrit LA, Deitsch KW, Scheidig C, Guinet F, Nehrbass U, Wellems TE, Scherf A, 2000. Frequent ectopic recombination of virulence factor genes in telomeric chromosome clusters of P. falciparum. Nature 407: 1018-1022.

9. Tibayrenc M, Ayala FJ, 1991. Towards a population genetics of micro-organisms: the clonal theory of parasitic protozoa. Parasitol Today 7: 228-232.

10. Babiker HA, Ranford-Cartwright LC, Currie D, Charlwood JD, Billingsley P, Teuscher T, Walliker D, 1994. Random mating in a natural population of the malaria parasite Plasmodium falciparum. Parasitology 109: 413-421.

11. Paul REL, Packer MJ, Walmsley M, Lagog M, RanfordCartwright LC, Paru R, Day KP, 1995. Mating patterns in malaria parasite populations of Papua New Guinea. Science 269: 1709-1711.

12. Snounou G, Zhu X, Siripoon N, Jarra W, Thaithong S, Brown KN, Viriyakosol S, 1999. Biased distribution of msp1 and msp2 allelic variants in Plasmodium falciparum populations in Thailand. Trans $R$ Soc Trop Med Hyg 93: 1-6.

13. Moelans IIMD, Klaassen CHW, Kaslow DC, Konings RNH, Schoenmakers JGG, 1991. Minimal variation in Pfs16, a novel protein located in the membrane of gametes and sporozoites of Plasmodium falciparum. Mol Biochem Parasitol 46: 311-314.

14. Baker DA, Daramola O, McCrossan MV, Harmer J, Targett GAT, 1994. Subcellular localisation of Pfs16, a Plasmodium falciparum gametocyte antigen. Parasitology 108: 129-137.

15. Favaloro JM, Coppel RL, Corcoran LM, Foote SJ, Brown GV, Anders RF, Kemp DJ, 1986. Structure of the RESA gene of Plasmodium falciparum. Nucleic Acids Res 14: 8265-8277.

16. Kyes S, Pinches R, Newbold C, 2000. A simple RNA analysis method shows var and rif multigene family expression patterns in Plasmodium falciparum. Mol Biochem Parasitol 105: 311315.

17. Jarra W, Snounou G, 1998. Only viable parasites are detected by PCR following clearance of rodent malarial infections by drug treatment or immune responses. Infect Immun 66: 3783-3787.

18. von Seidlein L, Milligan P, Pinder M, Bojang K, Anyalebechi C, Gosling R, Coleman R, Ude JI, Sadiq A, Duraisingh M, Warhurst D, Alloueche A, Targett G, McAdam K, Greenwood B, Walraven G, Olliaro P, Doherty T, 2000. Efficacy of artesunate plus pyrimethamine-sulphadoxine for uncomplicated malaria in Gambian children: a double-blind, randomised, controlled trial. Lancet 355: 352-357.

19. Janse CJ, 1993. Chromosome size polymorphism and DNA rearrangements in Plasmodium. Parasitol Today 9: 19-22.

20. Conway DJ, Roper C, Oduola AMJ, Arnot DE, Kremsner PG, Grpbusch MP, Curtis CF, Greenwood BM, 1999. High recombination rate in natural populations of Plasmodium falciparum. Proc Natl Acad Sci USA 96: 4506-4511.

21. Tami A, Grundmann H, Sutherland C, McBride J, Cavanagh D, Campos E, Snounou G, Barnabé C, Tibayrenc M, Warhurst D, 2002. Restricted genetic and antigenic diversity of Plasmodium falciparum under mesoendemic transmission in the Venezuelan Amazon. Parasitology: 124: 569-581.

22. Daubersies P, Sallenave-Salles S, Magne S, Trape J-F, Contamin H, Fandeur T, Rogier C, Mercereau-Puijalon O, Druilhe P, 1996. Rapid turnover of Plasmodium falciparum populations in asymptomatic individuals living in a high transmission area. Am J Trop Med Hyg 54: 18-26.

23. Contamin H, Fandeur T, Rogier C, Bonnefoy S, Konate L, Trape J-F, Mercereau-Puijalon O, 1996. Different genetic characteristics of Plasmodium falciparum isolates collected during successive malaria episodes in Senegalese children. Am J Trop Med Hyg 54: 632-643.

24. Färnert A, Snounou G, Rooth I, Bjorkman A, 1997. Daily dynamics of Plasmodium falciparum subpopulations in a holoendemic area. Am J Trop Med Hyg 56: 538-547.

25. Hayward RE, Tiwari B, Piper KP, Baruch DI, Day KP, 1999. Virulence and transmission success of the malarial parasite Plasmodium falciparum. Proc Natl Acad Sci USA 96: 45634568. 